\title{
A Study on Wave Run-up Height and Depression Depth around Air-water Interface-piercing Circular Cylinder
}

\author{
Bon-Guk Koo $^{* \dagger} \cdot$ Dong-Woo Park ${ }^{* *} \cdot$ Kwang-Jun Paik ${ }^{* * *}$ \\ * Department of Naval Architecture and Marine Engineering, Changwon National University, Changwon, Korea \\ ** Department of Naval Architecture and Ocean Engineering, Tongmyong University, Busan, Korea \\ *** Samsung Ship Model Basin, Samsung Heavy Industries, Daejeon, Korea
}

\begin{abstract}
In this paper, the wave run-up height and depression depth around air-water interface-piercing circular cylinder have been numerically studied. The Reynolds Averaged Navier-Stokes equations (RANS) and continuity equations are solved with Reynolds Stress model (RSM) and volume of fluid (VOF) method as turbulence model and free surface modeling, respectively. A commercial Computational Fluid Dynamics (CFD) software "Star-CCM+" has been used for the current simulations. Various Froude numbers ranged from 0.2 to 1.6 are used to investigate the change of air-water interface structures around the cylinder and experimental data and theoretical values by Bernoulli are compared. The present results showed a good agreement with other studies. Kelvin waves behind the cylinder were generated and its wave lengths are longer as Froude numbers increase and they have good agreement with theoretical values. And its angles are smaller with the increase of Froude numbers.
\end{abstract}

Key Words : CFD, Interface-piercing circular cylinder, Wave run-up, Depression depth, Free surface

\section{Introduction}

Recently, ocean and offshore industries have been paid attention remarkably due to the steep fuel prices caused by continuous increase of demand in energy. Those offshore structures such as semi-submersibles, drilling rigs, and tension leg platforms (TLP) are usually composed of circular or square cylinder columns as their hull. The flow around these blunt bodies is much more complicated than that of onshore structures due to the interaction between the viscous effects near the body and air-water interface phenomena, including wave run-up, wave depression, and breaking waves. Especially, the wave run-up is one of important design factors when offshore structures are operated and the minimum limit is usually required in owner's specifications.

There are several experimental and numerical studies on the wave run-up height/depression depth around surface-piercing circular cylinder. Hey (1947) measured the maximum heights of the bow waves in front of the circular cylinder and the depths of the depression on the center line behind cylinder at a wide range of Froude (Fr) numbers. Chaplin and Teigen (2003) carried out experimental study at a constant ratio of Reynolds (Re) number to Froude number of $2.79 \times 10^{5}$. They measured the run-up on the front of the cylinder and the depth of depression at the back.

† Corresponding Author : bonguk9@changwon.ac.kr, 055-213-3686
Flows past an interface piercing cylinder at various Reynolds and Froude numbers have been simulated by $\mathrm{Yu}$ et al. (2008), Suh et al. (2011), and Akira et al. (2013) using large eddy simulation (LES) and a volume of fluid (VOF) method. They showed that larger wave run-up heights and depression depths are observed with Fr increases. A truncated vertical circular cylinder in the regular wave is used to study the wave run-up height by Stanberg and Braaten (2002), Kristiansen et al. (2004), and Sung et al. (2007). Recently, Lee et al. (2013) studied the wave run-up phenomena experimentally and numerically with two different shapes of cylinder at various wave conditions.

In this paper, the wave run-up height and depression depth around the surface-piercing circular cylinder have been investigated using the commercial program. Various Froude numbers (0.2 - 1.6) are used to study the change of air-water interface around the cylinder. Experimental data and theoretical values by Bernoulli are compared with current wave run-up heights and depression depths.

\section{Computational method}

\subsection{Mathematical and numerical method}

All computations in the present study are performed using a commercial computational fluid dynamics (CFD) software "STAR $\mathrm{CCM}+$ " from CD-adapco. The flow is assumed to be governed by 


\section{A Study on Wave Run-up Height and Depression Depth around Air-water Interface-piercing Circular Cylinder}

the Reynolds averaged Navier-Stokes equations and the Reynolds Stresses Model (RSM) is used as a turbulent model. Thus, the governing equations such as continuity equation, three momentum equations, and two equations for the turbulent properties are solved. The continuity and the RANS equations for 3-dimensional incompressible turbulent flows can be expressed as follows:

$$
\begin{aligned}
& \frac{\partial U_{i}}{\partial x_{i}}=0 \\
& \begin{aligned}
\frac{\partial}{\partial t}\left(\rho U_{i}\right)+\frac{\partial}{\partial x_{i}}\left(\rho U_{i} U_{j}\right)= & -\frac{\partial p}{\partial x_{i}}+\frac{\partial}{\partial x_{j}}\left[\mu\left(\frac{\partial U_{i}}{\partial x_{j}}+\frac{\partial U_{j}}{\partial x_{i}}\right)\right] \\
& +\frac{\partial}{\partial x_{j}}\left(-\rho \overline{u_{i} u_{j}}\right)
\end{aligned}
\end{aligned}
$$

where, $U_{i}=(U, V, W)$ are velocity components in the direction of the Cartesian coordinate $x_{i}=(x, y, z), \mu$ is viscosity, $p$ is static pressure and $\left(-\rho \overline{u_{i} u_{j}}\right)$ is Reynolds stresses. The transportation equation of the Reynolds stresses can be expressed as follows:

$$
\begin{aligned}
& \frac{\partial}{\partial t}\left(\rho \overline{u_{i} u_{j}}\right)+\frac{\partial}{\partial x_{k}}\left(\rho U_{k} \overline{u_{i} u_{j}}\right) \\
& \left.=-\frac{\partial}{\partial x_{k}}\left[\left(\overline{u_{i} u_{j} u_{k}}\right)+\overline{P\left(\delta_{k j} u_{i}+\delta_{i k} u_{j}\right.}\right)+\frac{\partial}{\partial x_{k}}\left[\mu \frac{\partial}{\partial x_{k}} \overline{\left(u_{i} u_{j}\right.}\right)\right]-\rho\left(\overline{u_{i} u_{k}} \frac{\partial u_{i}}{\partial x_{k}}+\overline{u_{j} u_{k}} \frac{\partial u_{i}}{\partial x_{k}}\right) \\
& +\overline{\left(\frac{\partial u_{i}}{\partial x_{j}}+\frac{\partial u_{j}}{\partial x_{i}}\right)}-2 \mu\left(\frac{\partial u_{i}}{\partial x_{k}} \frac{\partial u_{i}}{\partial x_{k}}\right)
\end{aligned}
$$

The Volume of Fluid (VOF) function is applied to account for the air-water interface and allow for its arbitrary deformation. An additional equation for the VOF, $F$, is given as follows:

$$
\frac{D F}{D t}=\frac{\partial F}{\partial t}+U_{i} \frac{\partial F}{\partial x_{i}}=0
$$

Liquid and gas are considered as two immiscible components of a single effective fluid, whose properties are assumed to vary based on the volume fraction of each component as follows for the density $\rho$ and viscosity $\mu$ :

$$
\begin{aligned}
& \rho=\rho_{G} F+\rho_{L}(1-F) \\
& \mu=\mu_{G} F+\mu_{L}(1-F)
\end{aligned}
$$

The convection and diffusion terms are discretized by the 2nd order upwind scheme and the 2nd order central-difference scheme, respectively. The high resolution interface capturing scheme (HRIC) (Muzaferija et al. 1998) is used for the free-surface capturing. For the pressure-velocity coupling, the SIMPLE algorithm (Ferziger and Peric, 2003) is used.

\section{Simulation conditions}

In the present simulations, various Fr (from 0.2 to 1.6) were used to investigate the air-water interface structures. It is noted that a relatively small Reynolds number (200), compared to other studies $(27,000)$, is applied in order to focus on the free surface structures of the flow. These two non-dimensional numbers are defined as follows;

$$
F r=\frac{U_{\infty}}{\sqrt{g D}}, R e=\frac{U_{\infty} D}{\nu}
$$

where, $U_{\infty}$ is a uniform incoming velocity, $D$ is a cylinder diameter. In the commercial software, Re and Fr were considered using $g$ and $\nu$, respectively.

The computational domain size is $-5 \leq \mathrm{x} / \mathrm{D} \leq 25,-8 \leq \mathrm{y} / \mathrm{D} \leq$ 8 , and $-4 \leq \mathrm{z} / \mathrm{D} \leq 2$ with $0.89 \mathrm{M}$ grid points for $\operatorname{Fr}(=0.2-1.2)$ as shown in Fig. 1. For $\operatorname{Fr}(=1.4,1.6)$, the domain size is $-5 \leq x / D$ $\leq 40,-20 \leq \mathrm{y} / \mathrm{D} \leq 20$, and $-4 \leq \mathrm{z} / \mathrm{D} \leq 2$ with $1.5 \mathrm{M}$ grid points. Three different grids (coarse, medium, fine) were used to investigate the influence of the grid resolution. This grid study is performed with $\mathrm{Fr}=1.0$. The grid parameters are shown in Table 1. The medium grid is for the present simulations at up to $\mathrm{Fr}=1.2$. However, the fine grid is used at higher $\operatorname{Fr}(=1.4,1.6)$. Two volumetric meshes are applied on the near the cylinder body and air-water interface area in order to refine the meshes as shown in Fig. 1.

A velocity inlet boundary condition with a uniform inflow ( $\left.U_{\infty}=1\right)$ and a pressure outlet boundary conditions were applied. No-slip boundary conditions were applied on the cylinder wall, while the slip boundary condition was adopted at the side walls, the bottom, and the top of the computational domain as shown in Fig. 1 and $y+$ is about 30. A uniform velocity field as the upstream velocity is prescribed to the entire computational domain at rest. It is noted that a damping zone (half domain size from the cylinder to outlet) was used in order to avoid reflecting waves from the outlet boundary and more detail can be found in Choi and Yoon (2009). 


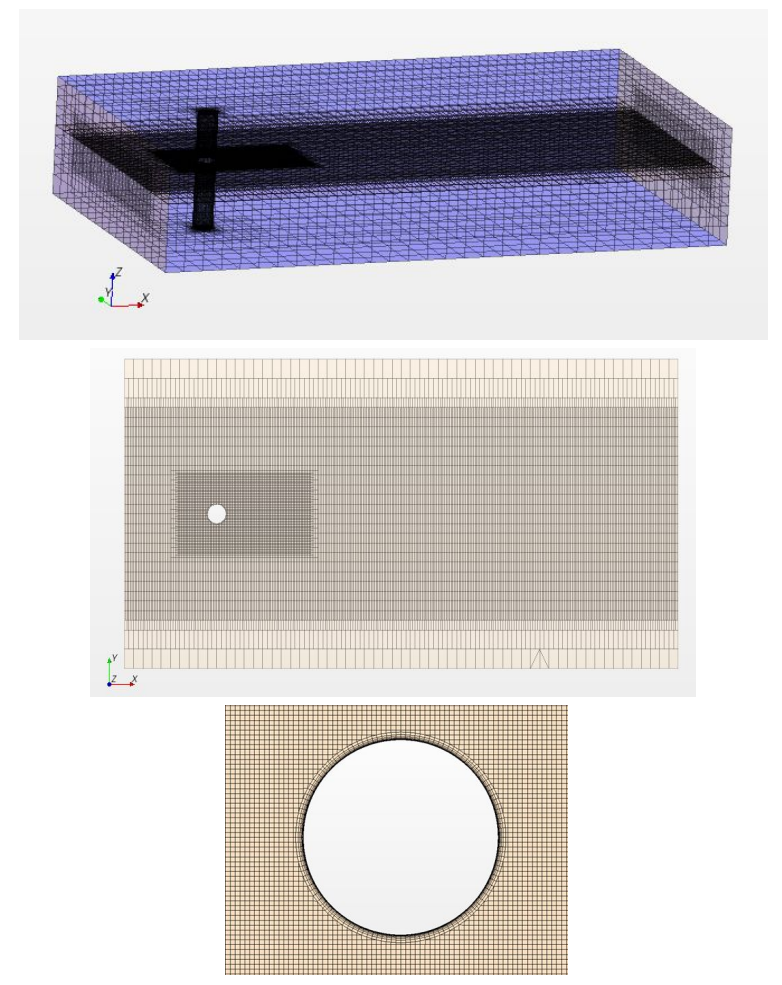

Fig. 1. Computational domain with grid.

Table 1. Grid and wave run-up height

\begin{tabular}{lll}
\hline Grid & Grid size & Run-up \\
\hline \hline Coarse & $0.6 \mathrm{M}$ & 0.282 \\
\hline Medium & $0.89 \mathrm{M}$ & 0.342 \\
\hline Fine & $1.5 \mathrm{M}$ & 0.341 \\
\hline Bernoulli $\left(\mathrm{Fr}^{2} / 2\right)$ & - & 0.32 \\
\hline M: Million & &
\end{tabular}

\section{Results}

\subsection{Flow near the interface (air-water interface structures)}

The instantaneous air-water interface around the circular cylinder are shown in Fig. 2. In Fig. 2, there are three different views from the left column to right column, front view, side view, and wake view, respectively. At smallest Fr $(=0.2)$, which is not shown in the present paper, the deformation of the free surface is negligibly small and no waves are generated in the wake region behind the cylinder. For $\operatorname{Fr}(=0.4,0.8)$, relatively smaller bow waves are observed in front of the cylinder with Kelvin waves behind the cylinder. Since Reynolds effects are neglected in this paper, there is hardly seen small air-water interface structures in the wake region behind the cylinder which is observed in high Reynolds simulations (Akira et al. (2013)). For Fr (=1.0 -1.4), much increased bow wave is observed in front of cylinder and it breaks and wraps around the bow. The depression exist on the downstream side of the cylinder. At highest Fr (=1.6), the bow wave increases remarkably with the largest wake region among different Fr and plunging wave breakers are observed around the cylinder. The depression depth also shows much deeper than those with other Fr.

The instantaneous air-water interface elevation contours shown in Fig. 3 and diverging and transverse wave pattern are observed for each Fr. The diverging waves are more dominant than the transverse waves with wave lengths that roughly correspond to the theoretical transverse wave length in a Kelvin wave pattern, i.e. $\lambda$ $=2 \pi \mathrm{Fr}^{2}$. The diverging wave lengths for all Fr agree well with theoretical values. It is noted that the same length scale is applied for the all $\mathrm{Fr}$ in order to compare each other. The diverging angle decreases linearly as Fr increases. The angles for all Fr are larger than that of the Kelvin wave value $\left(19^{\circ}\right)$ which is typically observed in ship flows. Bhushan et al. (2011) studied the flow around the air-cushion vehicle (ACV) numerically with different Froude numbers and they showed similar Kelvin wave angle patterns with current simulations.

\subsection{Wave run-up height and depression depth}

The run-up height is the maximum bow wave height in front of the cylinder and the depression depth is the largest cavity depth behind the cylinder. Iso-surface of free surface to indicate the run-up height and depression depth with different Fr numbers is shown in Fig. 4. The simulation with $\mathrm{Fr}=0.2$ is not presented in the Fig. 4 since the wave run-up height and depression depth are too small to identify. It is observed that both wave run-up heights and depression depths increase as Fr increases. Fig. 5 (a) presents the wave run-up height and depression depth at different Fr with other experimental and numerical studies. The wave run-up heights of the present simulations have good agreement with the Bernoulli's equation $\left(\mathrm{Fr}^{2} / 2\right)$ and the results from the previous studies by Chaplin and Teigen (2003), Yu et al. (2008), and Hey (1947). Although the previous studies used much different Re $(27,000)$, the free surface structures are fairly similar with current simulations.

The depression depth behind the cylinder for all $\mathrm{Fr}$ is shown in 
A Study on Wave Run-up Height and Depression Depth around Air-water Interface-piercing Circular Cylinder

(a)
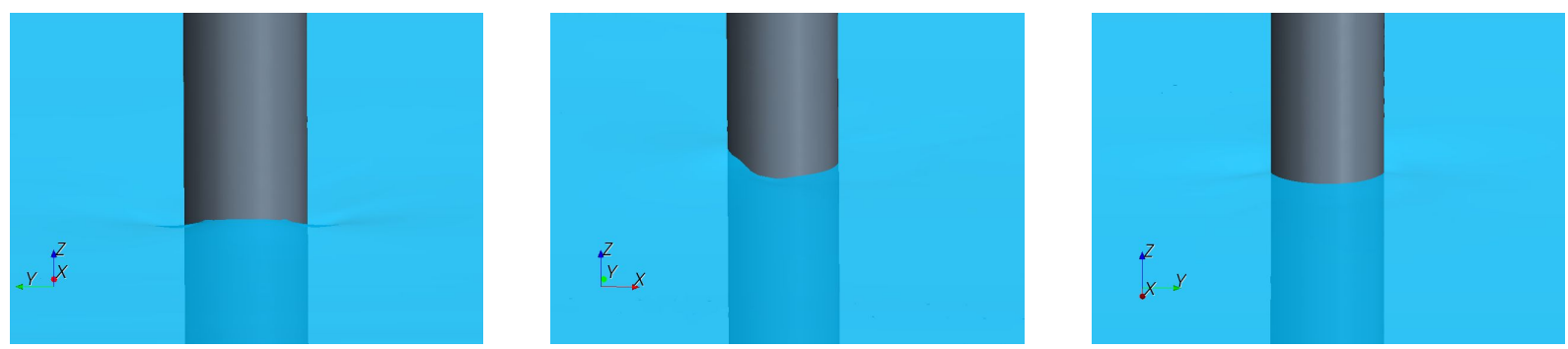

(b)
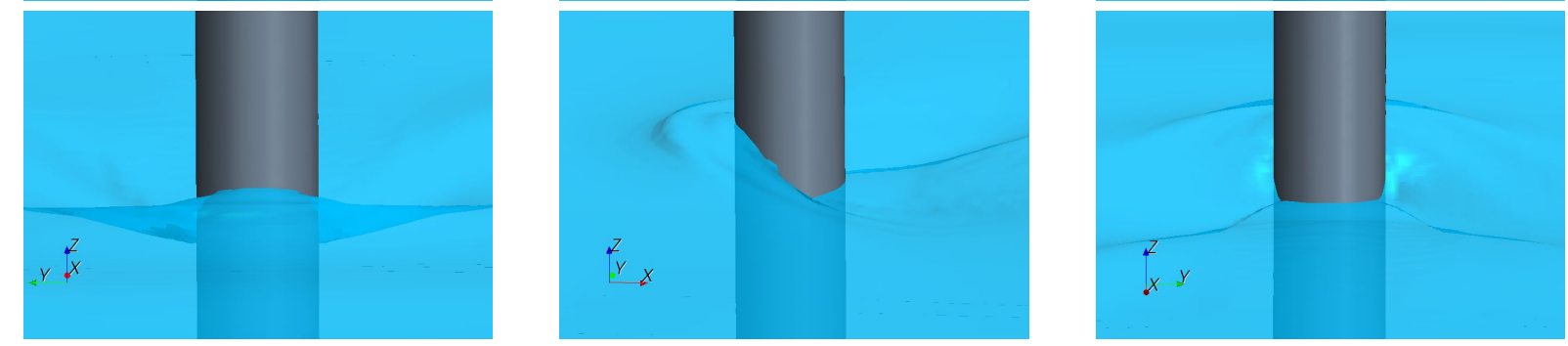

(c)
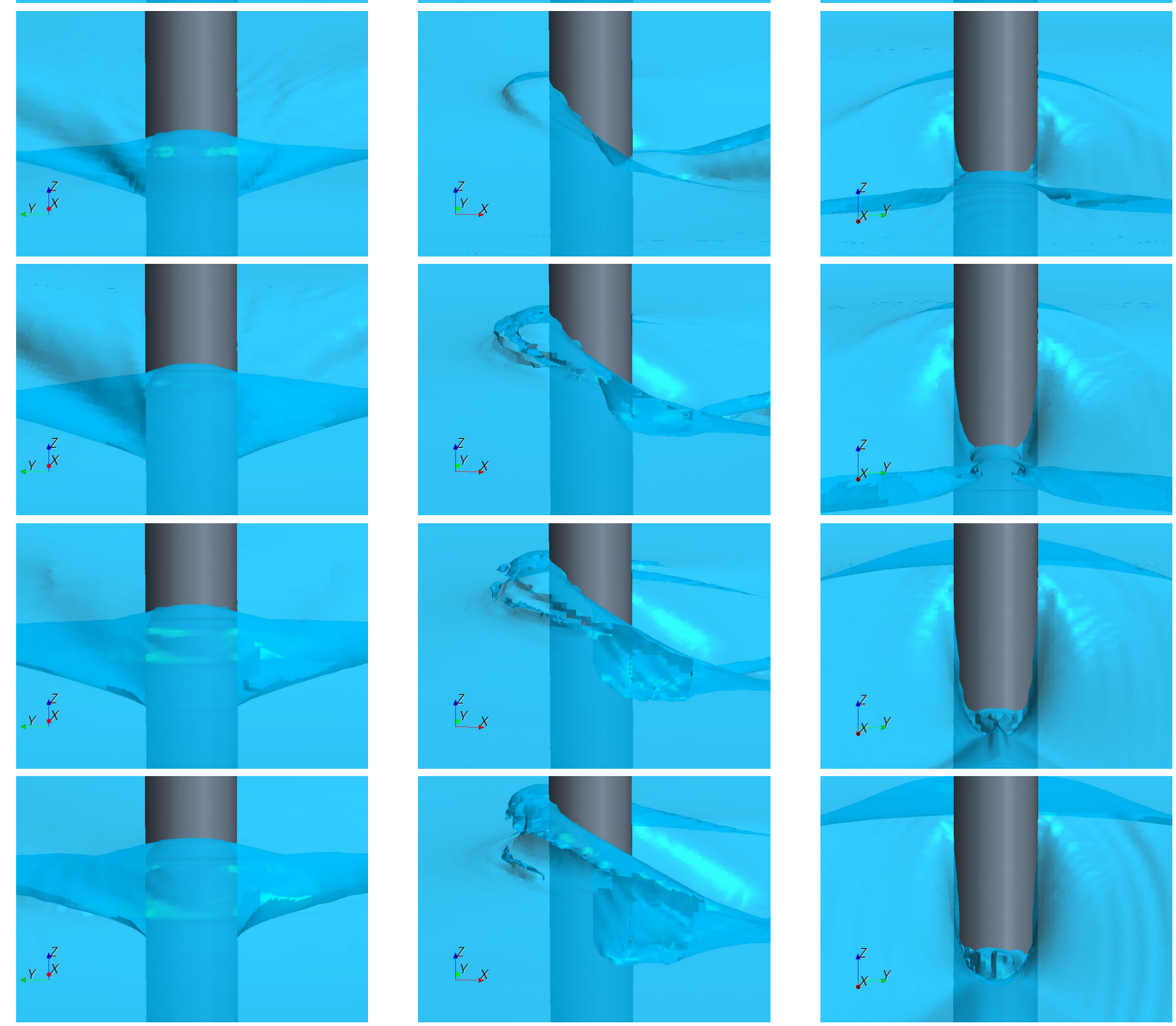

Fig. 2. Wave Field; (a) $\mathrm{Fr}=0.4$, (b) $\mathrm{Fr}=0.8$, (c) $\mathrm{Fr}=1.0$, (d) $\mathrm{Fr}=1.2$, (e) $\mathrm{Fr}=1.4$, (f) $\mathrm{Fr}=1.6$. $1^{\text {st }}$ column: front view, $2^{\text {nd }}$ column: side view, $3^{\text {rd }}$ column: wake view. 


\section{Bon-Guk Koo $\cdot$ Dong-Woo Park $\cdot$ Kwang-Jun Paik}

Fig. 5 (b). It is also compared with experimental study (Hey, 1947).

The current results predict in fairly good agreement with experimental data by Hey (1947). The cavity region behind the cylinder due to the depression makes the cylinder become different body shapes, i.e. its body changes into more slender such that the flow patterns around the cylinder changes significantly especially near the air-water interface. Further studies will be needed to investigate more details of this phenomena.
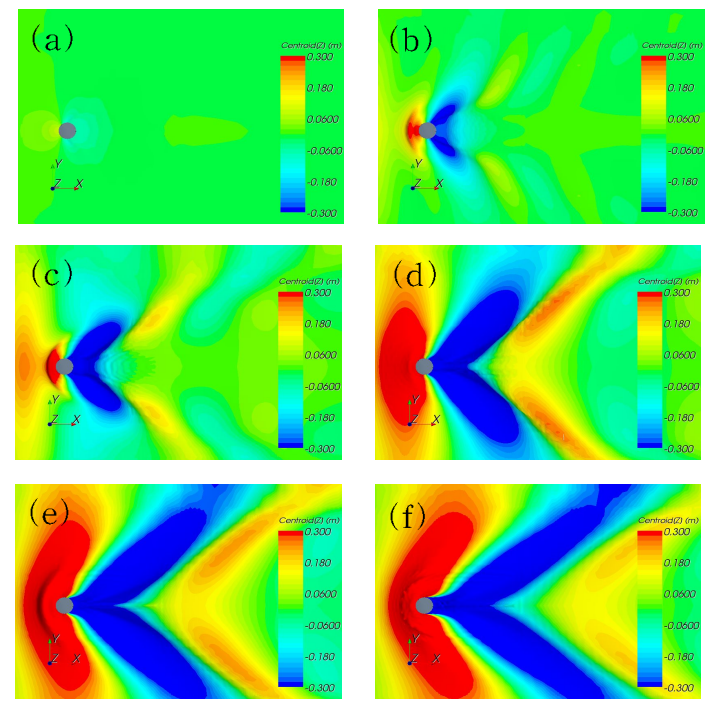

Fig. 3. Air-water interface elevation contours; (a) $\mathrm{Fr}=0.8$, (b) $\mathrm{Fr}=1.0$, (c) $\mathrm{Fr}=1.2$, (d) $\mathrm{Fr}=1.4$, (e) $\mathrm{Fr}=1.6$.
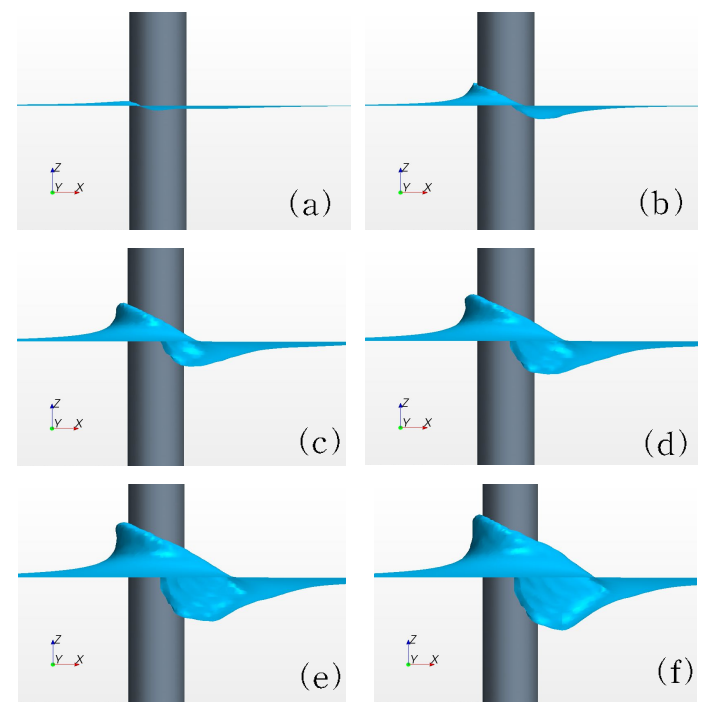

Fig. 4. Wave run-up height and depression depth; (a) $\mathrm{Fr}=0.4$, (b) $\mathrm{Fr}=0.8$, (c) $\mathrm{Fr}=1.0$, (d) $\mathrm{Fr}=1.2$, (e) $\mathrm{Fr}=1.4$, (f) $\mathrm{Fr}=1.6$. (a)

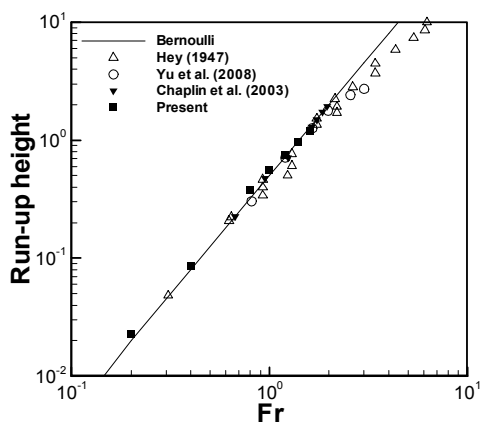

(b)

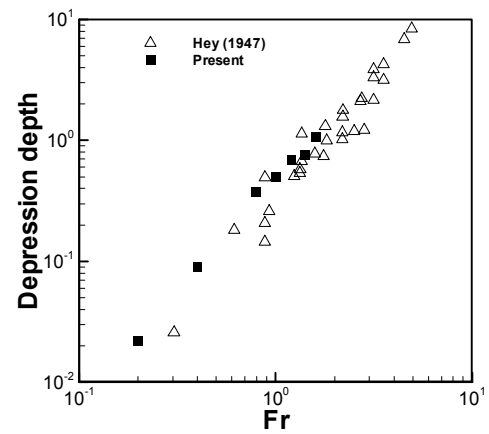

Fig. 5. Run-up height and depression depth. Re; Hey: 27,000, Yu et al. (2008): 27,000, Chaplin et al. (2003): upto 470,000 .

\section{Conclusions}

The wave run-up height and depression depth around air-water interface-piercing circular cylinder have been investigated using a commercial CFD program. Various Froude numbers $(0.2$ - 1.6) are used to study the change of air-water interface structures around the cylinder and experimental data and theoretical values by Bernoulli are compared with current wave run-up heights and depression depths. The current results are in fairly good agreement with other studies. Kelvin waves are generated behind the cylinder and its wave lengths are longer as Fr increase and they match well with theoretical values. Its angles are smaller with the increase of Fr.

As future work, various wave conditions will be applied to get wave run-up height in front of the cylinder for the practical design of offshore structures in the real shipyards.

\section{Acknowledgements}

This paper was supported by research funds of Changwon National University in 2014. 
A Study on Wave Run-up Height and Depression Depth around Air-water Interface-piercing Circular Cylinder

\section{References}

[1] Akira, H., J. Yang and F. Stern(2013), Toward Wall Modeling in Cartesian Grid Solver Using Overset Grid Technique for Ship Hydrodynamics. Proceedings of the ASME 2013 Fluids Engineering Division Summer Meeting FEDSM2013. Incline Village, Nevada, USA, pp. 1-7.

[2] Bhushan, S., F. Stern and L. J. Doctors(2011), Verification and validation of URANS wave resistance for air cushion vehicles, and comparison with linear theory. Journal of Ship Research, Vol. 55, No. 4, pp. 249-267.

[3] Chaplin, J. R. and P. Teigen(2003), Steady Flow past a Vertical Surface-Piercing Circular Cylinder. Journal of Fluids and Structures, Vol. 18, pp. 271-285.

[4] Choi, J. and S. Yoon(2009), Numerical Simulation Using Momentum Source Wave-maker applied to RANS Equation Model. Coastal Engineering, Vol. 56, pp. 1043-1060.

[5] Ferziger, J. H. and M. Peric(2003), Computational Methods for Fluid Dynamics. Springer, Berlin, $3^{\text {rd }}$ edition.

[6] Hey, A. D.(1947), Flow about Semi-Submerged Cylinders of Finite Length, Princeton University Report.

[7] Lee, S., S. Han, Y. Choi, S. Kwon, D. Jung and S. Park(2013), Study on Wave Run-Up Phenomenon over Vertical Cylinder. Journal of Ocean Engineering and Technology, Vol. 27, No. 4, pp. 62-67.

[8] Muzaferija, S., M. Peric, P. Sames and T. Schellin(1998), A Two-Fluid Navier-Stokes Solver to Simulate Water Entry. In Proc 22nd Symposium Naval Hydrodynamics, Washington, DC, USA, pp. 277-289.

[9] Kristiansen, T., R. Baarholm and C. T. Stansberg(2004), Validation of Second-order Analysis in Predicting Diffracted Wave Elevation around a Vertical Circular Cylinder. Proc. 14th ISOPE Conf., pp. 342-349.

[10] Stansberg, C. T. and H. Braaten(2002), Nonlinear Wave Disturbance around a Vertical Cylinder Column. Proc. 21st OMAE Conf., pp. 1-7.

[11] Suh, J., J. Yang and F. Stern(2011), The Effect of Air-Water Interface on the Vortex Shedding from a Vertical Circular Cylinder. Journal of Fluids and Structures, Vol. 27, pp. 1-22.

[12] Sung, H. G., Y. S. Kim, B. W. Nam and S. Y. Hong(2007), Experimental Investigation of Wave Loads on a Truncated Vertical Circular Cylinder. Proceedings of The Korean Society of Ocean Engineering Conference, pp. 105-111.

[13] Yu, G., E. J. Avital and J. J. R. Williams(2008), Large Eddy
Simulation of Flow Past Free Surface Piercing Circular Cylinders. Journal of Fluids Engineering, Vol. 130, pp. 101304.1-101304.9.

Received : 2014. 05. 16.

Revised : 2014. 06. 18

Accepted : 2014. 06. 25. 